\title{
EDITORIAL
}

\section{Pharmacogenomics: a key component of personalized therapy}

\author{
Matthias Schwab ${ }^{1,2 *}$ and Elke Schaeffeler ${ }^{1,2}$
}

The goal of personalized medicine is to provide individualized treatment and to predict the clinical outcome of different treatments in different patients. Pharmacogenomics is one of the core elements in personalized medicine. The basic concept is that interindividual variability in drug response is a consequence of multiple factors, including genomics, epigenomics, the environment and a patient's characteristics, such as gender, age and/or concomitant medication [1]. Thirty years ago, drug response was found to be altered by genetic polymorphisms in drug-metabolizing enzymes (for example, the cytochrome P450 2D6 and the thiopurine S-methyltransferase) [2], yet valid and predictive biomarkers for therapeutic effects and/or for avoiding severe side effects are lacking for more than $90 \%$ of drugs currently used in clinical practice. Pharmacogenomics in recent years has used a new generation of technologies known as 'omics' approaches that has led to a revolution in the understanding of disease susceptibility and pathophysiology, providing enormous potential for novel therapeutic strategies.

It is beyond doubt that pharmacogenomics promotes the development of targeted therapies, as was demonstrated by the approval earlier this year of the drug ivacaftor by the US Food and Drug Administration (FDA) and the European Medicines Agency for the treatment of a subset of cystic fibrosis patients. Ivacaftor is approved only for cystic fibrosis patients bearing the specific G551D genetic variant in the cystic fibrosis transmembrane regulator $(C F T R)$ gene, which encodes a protein that regulates chloride and water transport in the body and is defective in the disease. Ivacaftor targets the CFTR protein, increases its activity, and consequently improves lung function [3].

Although this and other examples (such as vemurafenib as an inhibitor of the BRAF V600E mutation in malignant

\footnotetext{
*Correspondence: matthias.schwab@ikp-stuttgart.de

'Dr Margarete Fischer-Bosch-Institute of Clinical Pharmacology, Auerbachstrasse

112, 70376, Stuttgart, Germany

Full list of author information is available at the end of the article
}

melanoma [1]) suggest the demise of the blockbuster model of drug development, the concept of targeted therapy is in its early stages. One reason is that monogenic pharmacogenetic traits are mostly unable to explain the variations in a complex phenotype such as drug response [2]. There is evidence through drug-target network analyses that most currently used drugs have multiple targets and numerous off-target effects. Genome-wide approaches such as sequencing, epigenomic profiling and metabolomics will be essential for understanding the detailed molecular architecture of disease etiology and/ or drug response. Genome-wide association studies (GWAS) have implicated many new biological pathways, but this approach has limitations because most of the variants that have been associated with clinical phenotypes, such as adverse drug reactions, are not necessarily causal.

There is reasonable hope that pharmacogenomic research will benefit from a combination of different omics technologies. Recently, multi-omics studies have shown their use in discovering potential novel therapeutic targets [1]. For instance, in one multi-omics study the integrative personal omics profile (iPOP), which combines genomic information with additional dynamic omics activities (that is, transcriptomic, proteomic, metabolomic and autoantibody profiles), from a single individual over a 14-month period demonstrated that iPOP data can be used to interpret healthy and diseased states, and can be helpful in the diagnostics, monitoring and treatment of diseased states [4].

The major challenge, however, is the bioinformatic analysis and valid interpretation of highly complex multiomics data sets. A recent National Institutes of Health White Paper by the Quantitative and Systems Pharmacology Workshop Group [5] stated that: 'Genomics is, in and of itself, insufficient as a means to develop and study drugs: the operation of biological networks is strongly affected not only by changes in coding sequence or gene expression but also by transient responses to external signals at the level of protein activity, posttranslational modification, stochastic processes, etc.' Thus, with the help of an integrative systems pharmacology approach, multiple one-dimensional biomolecular-omics data sets, 
as well as patient history, can be linked together to achieve a better understanding of the biology behind diseases as well as drug-response phenotypes. Such a strategy should ultimately result in the identification of novel drug targets.

Several important applications of pharmacogenomics are already being used in clinical practice and some of them have been approved by the FDA (for example, cetuximab/panitumumab and $K R A S$; vemurafenib and $B R A F$; warfarin and CYP2C9/VKORC1; abacavir and $H L A-B * 5701$; carbamazepin and $H L A-B * 1502$; thiopurines and TPMT) [1]. Other candidates have been identified (for example, tamoxifen [6]), but their clinical utility needs to be evaluated. To improve the translation of pharmacogenomics from bench to bedside, the dynamic relationship between a patient's phenotype (such as drug response), which may change over time, and their genome also needs to be more deeply considered (Figure 1). The integration of non-genetic factors, such as environmental and clinical co-variates, may provide important additional phenotypic information to increase the precision of a therapeutic decision, as recently shown by warfarin algorithms [7]. In addition to genetic variation in CYP2C9 and VKORC1, warfarin dose requirement depends on age, sex, body mass index, diet, concomitant drug therapy and ethnic background. The consideration of all these co-variates predicts up to $60 \%$ of the variability of warfarin dosage in patients. Consequently, warfarin pharmacogenomics treatment algorithms incorporating genetic and non-genetic factors have been established, extensively validated [8] and are now publicly available via the Internet [9].

There has been considerable research into pharmacogenomics in the past decade, and functional genomic approaches are likely to be used in the future as an important resource for the prediction of clinical outcome. However, the field faces a major challenge: how can pharmacogenomics knowledge be brought to the bedside as a key component of personalized medicine? In this context, electronic medical records (EMRs) and electronic health records (EHRs) may play a pivotal role. Information management and analysis of the clinical relevance of pharmacogenomics can be improved by using EMRs [10]. EMRs will help to compare data on treatment and outcome in thousands of patients using a real clinical setting, including the integration of genomic and multi-omics data. Fitting EMRs/EHRs into a dynamic, validated and rapidly evolving information infrastructure [11] is also crucial for pharmacogenomics.

Without doubt, pharmacogenomics is a highly attractive field of research, which has been recently stimulated by multi-omics technologies. To demonstrate the clinical relevance of pharmacogenomics in most areas of medicine, however, a concerted effort is necessary to connect the

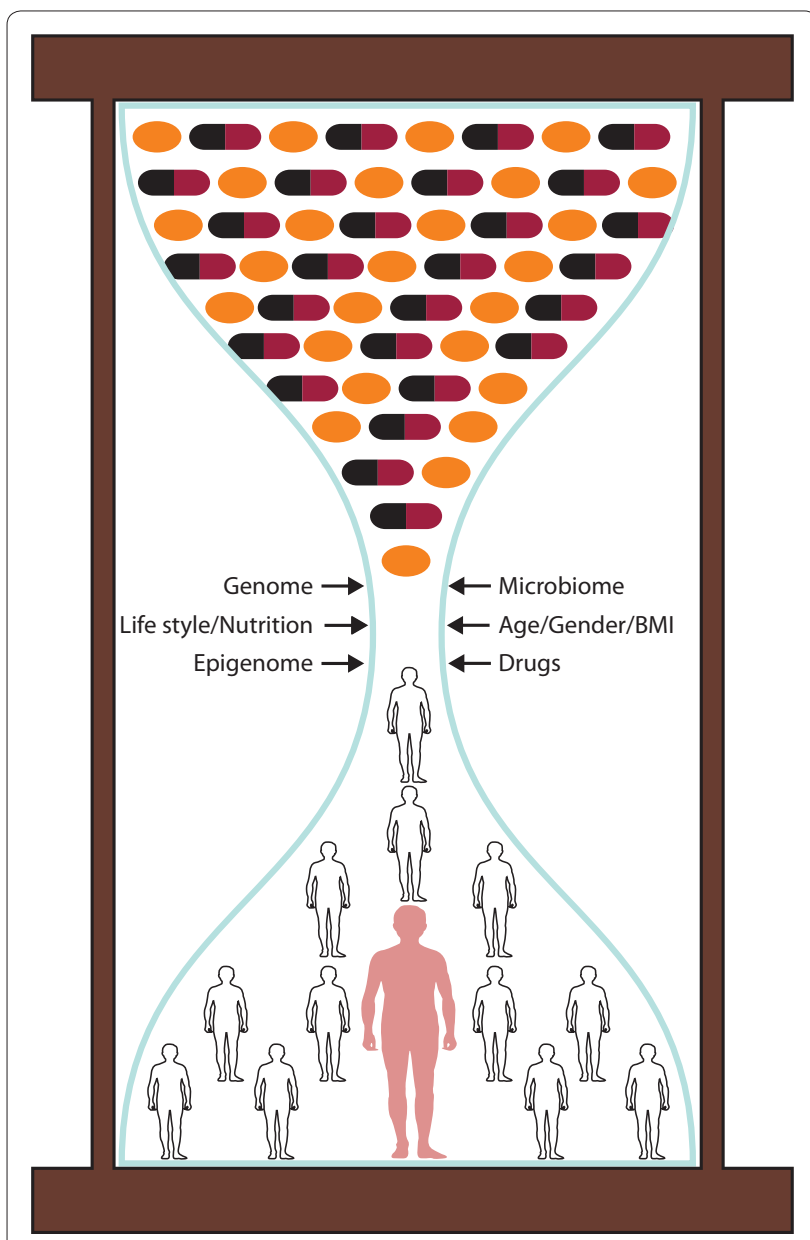

Figure 1. Pharmacogenomics. Interindividual variation in drug response is the consequence of a combination of genetic and environmental factors as well as patient characteristics, which affect the pharmacokinetics and/or pharmacodynamics of drugs. Pharmacogenomics affects not only therapeutic efficacy but also disease susceptibility and drug development. BMI, body mass index.

expertise of basic and clinical researchers with other sectors such as healthcare communities, regulators and commercial partners.

\section{Abbreviations}

EHR, electronic health record; EMR, electronic medical record; iPOP, integrative personal omics profile.

\section{Competing interests}

The authors declare that they have no competing interests.

\section{Acknowledgements}

The authors are supported by the German Federal Ministry of Education and Research (BMBF Virtual Liver grant 0315755 and grant 03 IS 2061C) the German Research Organisation (DFG SCHW 858/1-1), the FP7 EU Initial Training Network"Fighting Drug Failure" (PITN-GA-2009-238132), and the Robert Bosch Foundation in Stuttgart, Germany.

\section{Author details}

'Dr Margarete Fischer-Bosch-Institute of Clinical Pharmacology, Auerbachstrasse 112, 70376, Stuttgart, Germany. ${ }^{2}$ Department of Clinical Pharmacology, University Hospital, 72076 Tuebingen, Germany. 
Published: 29 November 2012

\section{References}

1. Meyer UA, Zanger UM, Schwab M: Omics and drug response. Annu Rev Pharmacol Toxicol 2012. doi: 10.1146/annurev-pharmtox-010510-100502.

2. Kalow W: Pharmacogenetics and pharmacogenomics: origin, status, and the hope for personalized medicine. Pharmacogenomics J 2006, 6:162-165.

3. Eckford PD, Li C, Ramjeesingh M, Bear CE: CFTR potentiator VX-770 (ivacaftor) opens the defective channel gate of mutant CFTR in a phosphorylation-dependent but ATP-independent manner. J Bio/ Chem 2012, 287:36639-36649.

4. Chen R, Mias Gl, Li-Pook-Than J, Jiang L, Lam HY, Chen R, Miriami E, Karczewski KJ, Hariharan M, Dewey FE, Cheng Y, Clark MJ, Im H, Habegger L, Balasubramanian S, O'Huallachain M, Dudley JT, Hillenmeyer S, Haraksingh R, Sharon D, Euskirchen G, Lacroute P, Bettinger K, Boyle AP, Kasowski M, Grubert F, Seki S, Garcia M, Whirl-Carrillo M, Gallardo M, et al:: Personal omics profiling reveals dynamic molecular and medical phenotypes. Cell 2012, 148:1293-1307.

5. Ward R (Ed): Quantitative and Systems Pharmacology in the Post-genomic Era: New Approaches to Discovering Drugs and Understanding

Therapeutic Mechanisms. [http://www.nigms.nih.gov/nr/ rdonlyres/8ecb1f7c-be3b-431f-89e6-a43411811ab1/0/ systemspharmawpsorger2011.pdf]
6. Brauch H, Schroth W, Goetz MP, Mürdter TE, Winter S, Ingle JN, Schwab M, Eichelbaum M: Tamoxifen use in postmenopausal breast cancer: CYP2D6 matters. J Clin Oncol 2012. doi: 10.1200/JCO.2012.44.6625.

7. Johnson JA, Gong L, Whirl-Carrillo M, Gage BF, Scott SA, Stein CM, Anderson JL, Kimmel SE, Lee MT, Pirmohamed M, Wadelius M, Klein TE, Altman RB, Clinical Pharmacogenetics Implementation Consortium: Clinical Pharmacogenetics Implementation Consortium Guidelines for CYP2C9 and VKORC1 genotypes and warfarin dosing. Clin Pharmacol Ther 2011, 90:625-629.

8. Schwab M, Schaeffeler E: Warfarin pharmacogenetics meets clinical use Blood 2011, 18:2938-2939.

9. WarfarinDosing [http://www.warfarindosing.org]

10. Wilke RA, Xu H, Denny JC, Roden DM, Krauss RM, McCarty CA, Davis RL, Skaar T, Lamba J, Savova G: The emerging role of electronic medical records in pharmacogenomics. Clin Pharmacol Ther 2011, 89:379-386.

11. Mandl KD, Kohane IS: Escaping the EHR trap--the future of health IT. N Engl J Med 2012, 366:2240-2242.

doi:10.1186/gm394

Cite this article as: Schwab M, Schaeffeler E: Pharmacogenomics: a key component of personalized therapy. Genome Medicine 2012, 4:93. 